\title{
Osteopoikilosis - a case report
}

\author{
Alexandra Rusu ${ }^{1}$, Anca Cardoneanu ${ }^{1,2}$, Alexandra Burlui ${ }^{1,2}$, Elena Rezus ${ }^{*, 1,2}$ \\ 1 "Grigore T. Popa" University of Medicine and Pharmacy, lasi, Romania, ${ }^{2}$ Rheumatology Department, \\ Rehabilitation Hospital lasi, Romania
}

\begin{abstract}
Osteopoikilosis (OPK) is a very rare and primarily benign autosomal dominant disorder of unknown etiology. Genetic studies showed heterozygous mutations of the LEMD3 gene. It is characterised by the occurrence of hyperostotic spots throughout the skeleton, with the most frequent localizations in the epiphyses and metaphyses of long bones, as well as in the carpal and tarsal bones. The clinical features of osteopoikilosis are poorly represented; consequently the condition is usually diagnosed incidentally on radiological examination or due to the association with other medical disorders (articular pain, fractures, joint dislocations, etc.). We report a case of osteopoikilosis in a 44 year-old man who presented with left hip joint pain and low back pain irradiated in left leg. Radiographs showed typical features of osteopoikilosis. Necessary investigations were done to exclude osteoblastic metastasis. The patient was treated with drugs and physiotherapy with significant improvement of the symptoms.
\end{abstract}

Keywords: osteopoikilosis, osteopathia condensans disseminate, Buschke-Ollendorf syndrome, overlap syndrome, sclerotic bone metastases

\section{Introduction}

Osteopoikilosis (OPK) is a benign rare disorder of the endochondral bone, characterized by small multiple osteocondensation lesions, maximum $10 \mathrm{~mm}$, usually found in the long bones of the limbs, hand, foot, pelvis and spine. The lesions are symmetrically distributed. Often, the diagnosis is made by chance, like in our case.

\section{Case Report}

A 44 year-old male from Targu Neamt is admitted for the first time in the $1^{\text {st }}$ Rheumatology Clinic of Rehabilitation Hospital

Received: May 2015; Accepted after review: June 2015; Published: June 2015.

${ }^{*}$ Corresponding author: Elena Rezuş, MD, PhD, Rheumatology Department, Rehabilitation Hospital, lasi, Associate Professor "Grigore T. Popa" University of Medicine and Pharmacy, lasi, Romania.

Email: elena rezus@yahoo.com from lasi complaining of mechanical pain in the left hip joint and lumbar rachialgia that irradiates in the left lower limb (on posterior thigh and calf). From his personal history, we discovered an incipient bilateral coxarthrosis, lumbar discopathy and an observation of osteopoikilosis, diagnosis which was suspected after a MRI a month before presentation.

The MRI showed small multiple osteocondensation lesions (intense hyposignal in T1 and T2 sections) at the level of proximal bilateral femural bone marrow, acetabulum and pelvis, with a maximum diameter of 11 $\mathrm{mm}$; the prostate had a modified signal, with an irregular prostatic capsule. These aspects impose differential diagnosis between osteopoikilosis and bone metastasis from a prostatic cancer, reason why the patient was sent into our clinic for further investigations.

The objective clinical examination revealed a static and dynamic lumbar syndrome and a left $S 1$ radicular syndrome (algic and paresthesic radicular syndrome). 
Laboratory tests were within normal limits. The prostate ultrasound and PSA were in normal limits.

X-Ray made on lumbar spine, hands, feet and pelvis showed: multiple osteosclerotic oval-round lesions with a maximum diameter of $10 \mathrm{~mm}$, without osteolysis, with a benign character and with a periarticular localization at the level of the fist, metacarpophalangeal

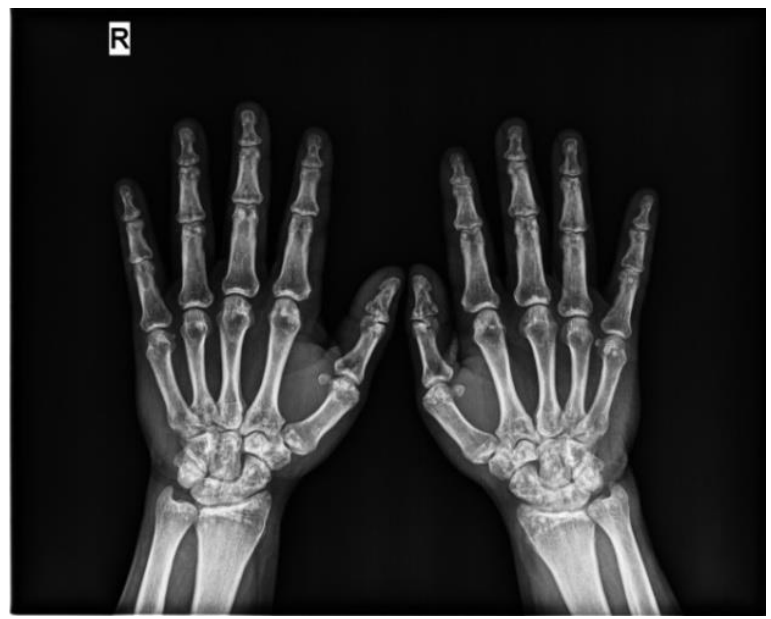

Fig. 1. Hand X-Ray

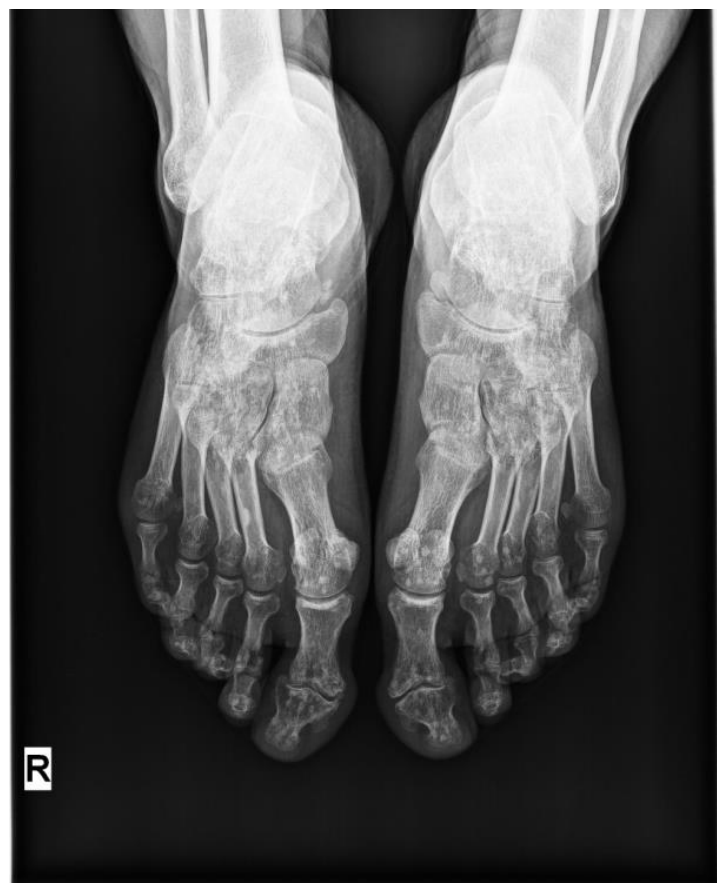

Fig. 2. Feet X-Ray joints, proximal interphalangeal joints, coxofemoral joints, peritarsal, metatarsophalangeal and interphalangeal joints. The lesions have a typical aspect of osteopoikilosis: symetrically and bilaterally distributed (Figures 1, 2 and 3). The lombar XRay shows discarthrosis at L3-L4 and L4-L5 levels (Figure 4).

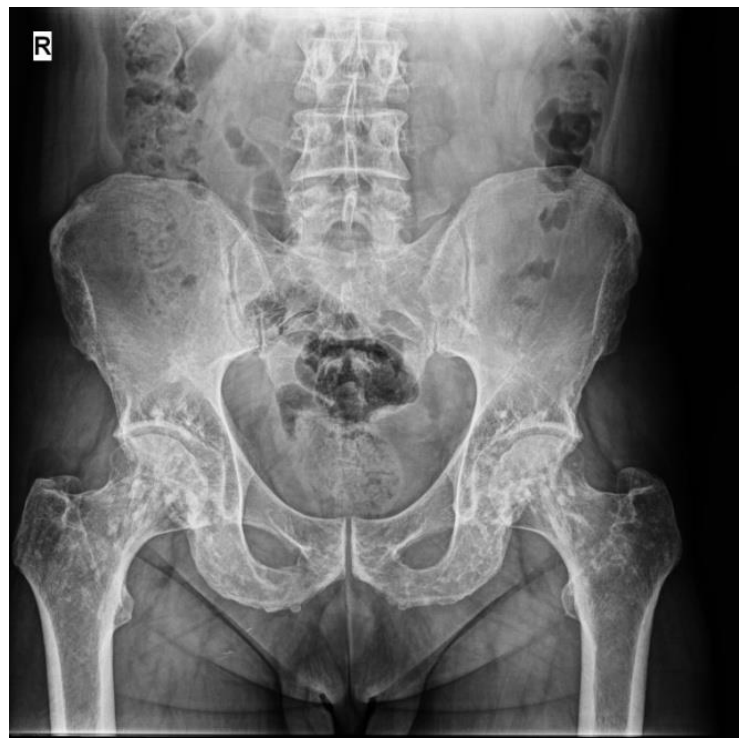

Fig. 3. Pelvis X-Ray

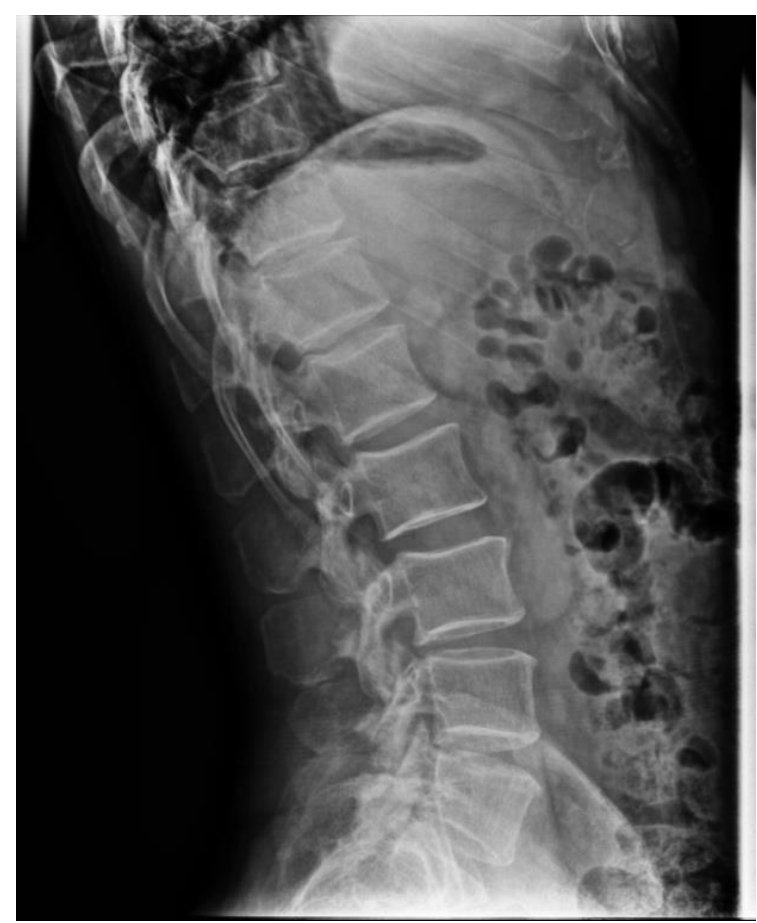

Fig. 4. Lombar spine X-Ray 
The clinical and paraclinical tests excluded the diagnosis of bone metastasis. Thus, the final diagnoses were: left $\mathrm{S} 1$ algic and paresthesic lomboradiculopathy due to discarthrosis, incipient bilateral coxarthrosis, and osteopoikilosis. Our patient does not present any clinical complications due to OPK. The disease is associated with a spine superposed discopathy.

During hospitalization, the patient received nonsteroidal anti-inflammatory drugs, neurotrophics and physiokinetotherapy, with an improvement of the symptomatology. He was discharged with the recommendation of using neurotrophics and chondroprotective agents and coming back for a reexamination after 6-8 months. To deepen the case, it is necessary to make $X$-Rays in patient's relatives and to follow the disease evolution of our patient.

\section{Discussions}

Osteopoikilosis or disseminated condensant osteopathy (Latin name) is a rare, benign bone dysplasia, with an unclear etiology and pathogenesis. It can appear sporadically [1, 12], but the up to date studies found a mutation in the LEMD1 gene (named also MAN1) that can be involved in the pathology of this disease [2-5]. Some authors consider OPK as a consequence of a sclerosant or a fibroproliferative disorder [6]. Women and men tend to be equally affected [7, 12], even though some reported cases showed a slightly increased prevalence for men $[8,10]$.

The disease prevalence is $1 / 10.000 .000$ residents; the incidence is $1 / 50.000$, but there are no recent data regarding this fact [11].

The first reference in the literature about osteopoikilosis dates from 1905, written by Stieda [9, 14]; the main characteristics were described in 1915 by Albers-Schonberg, a German radiologist who also described osteoporosis [8, 10].

Histologically, the disease is characterized by an abnormal maturation process of the endochondral bone. This process determines the appearance of osteocondensant lesions. Histologically, these lesions are made from dense trabeculae of the spongious bone, sometimes forming a nidus that cannot communicate with the bone marrow [12].

The diagnosis is strictly radiological, in most of the cases being made incidentally. The radiological characteristics of the OPK are: numerous oval-round densified lesions between 2 to $10 \mathrm{~mm}$, symmetrically distributed on the metaphysis and epiphysis of the long bones. These lesions persist in the childhood and during the lifelong [12, 13, 15]. On MRI, the lesions appear like hyposignal in $\mathrm{T} 1$ and T2 sections [7]. These lesions can increase or decrease in number and size and sometimes can disappear $[1,12,16]$. The main places for osteopoikilosis are the limb bones, the carpal and metacarpal bones, also tarsal and metatarsal bones. Rarely, it affects the pelvis, the spine, the ribs, the clavicles and the skull (very rarely) $[1,17]$.

Usually, the patients are asymptomatic, only $1 / 4$ of them having mild joint pain and joint effusion. There have been reported some disorders associated with OPK, like rheumatoid arthritis, synovial chondromatosis, ankylosing spondylitis [18].

Frequently, the disease is associated with dermatological disorders like: disseminated lenticular dermatofibrosis, keloid or discoid lupus and similar lesions to scleroderma [13, $14,19,20,30]$. Disseminated lenticular dermatofibrosis, a connective tissue disease associated with pigmented nevi, in combination with OPK, leads to BuschkeOllendorff syndrome [8]. Osteopoikilosis had also been associated with nanism, spinal stenosis, dystocia [30], organ anomanies such douplicated ureter, aorta coarctation, endocrine disorders, disc protrusions, melorheostosis and tuberous sclerosis [22, 28]. In the absence of skeletal or dermatological anomalies, OPK has been rarely reported in the literature. In romanian literature, the first case of OPK has been described in 2009 [23].

Concerning the presented case, we didn't observed any dermatological or organ anomalies; we found only the association with lumbar discarthrosis.

The differential diagnosis of osteopoikilosis includes: sclerotic bone metastasis (especially from prostate cancer) [21], osteopathia striata, 
osteoporosis, melorheostosis, tuberous sclerosis, hyperparathyroidism [27, 28], mastocytosis, enostosis, osteoma [14].

The major clinical problem is the confusion of OPK with metastatic osteoblastic lesions. The symmetric distribution, the predilect distribution in the metaphysis and epiphysis of long bones and the uniformity of the lesions are characteristics that suggest osteopoikilosis. Diagnosis is also sustained by normal values of acid and alkaline phosphatase and by scintigraphy [29]. The recognition of this disease (clinically asymptomatic and symmetric distribution of lesions on incidental X-Rays) makes other expensive investigations unuseful [1].

Bone scintigraphy reveals a slightly increase activity similar to enostosis bone isles, that reflects an active bone remodeling, especially in young patients with classical XRay findings that are representative for OPK. However, an abnormal scintigraphy image found in older patients should be highly investigated because, sometimes, osteopoikilosis can mask a bone metastasis

\section{References}

1. Resnick D, Niwayama G. Enostosis, hyperostosis and periostitis. Diagnosis of Bone and Joint Disorders. W.B Saunders Company, Philadelphia. 1988; 4084-4088.

2. Mumm S, Wenkert D, Zhang X, McAlister WH, Mier RJ, Whyte MP. Deactivating Germline Mutations in LEMD3 Cause Osteopoikilosis and Buschke-Ollendorff Syndrome, but Not Sporadic Melorheostosis. J Bone Miner Res 2007; 22(2):243-250.

3. Lin F, Morrison JM, Wu W, Worman HJ. MAN1, an integral protein of the inner nuclear membrane, binds Smad2 and Smad3 and antagonizes transforming growth factor-beta signaling. Hum Mol Genet 2000; 14:437-445.

4. Osada S, Ohmori SY, Taira M. XMAN1, an inner nuclear membrane protein, antagonizes BMP signaling by interacting with Smad1 in Xenopus embryos. Development 2003; 130:1783-1794.

5. Hellemans J, Preobrazhenska O. Willaert A et al. Loss-of-function mutations in LEMD3 result in osteopoikilosis,Buschke-Ollendorff syndrome and melorheostosis. Nat Genet 2004; 36:12131218.
[24]. A malign transformation of OPK lesions it is possible if cellular activity is present $[16,29]$. In literature, osteosarcoma and condrosarcoma have been reported [25, 27].

The overlap syndrome, known as mixed sclerosing bone dystrophy, combines OPK with other osteosclerotic bone disorders and it should be taken into consideration as a differential diagnosis. The combination between osteopoikilosis, melorheostosis and osteopathia striata (type 1) is the most frequent form of overlap syndrome [13, 26]. Osteopoikilosis doesn't require a proper treatment, only a periodical follow up.

\section{Conclusions}

Even if the natural course of this disease is benign and doesn't require a certain treatment, the complications and the coexisting pathological conditions demand doctor's attention. Therefore, it is important to perform a proper diagnosis concerning the rarity of this disease.

6. Kaparov A, Sari H, Akarirmark U, Uludag M. De Quervain's syndrome associated with osteopoikilosis: a case report and review of the literature. Rheumatol Int 2011; 31(6):809-813.

7. Khot R, Sikarwar JS, Gupta RP, Sharma GL. Osteopoikilosis: a case report. Ind J Radiol Imag 2005; 15(4):453-454.

8. Roberts NM, Langtry JAA, Branfoot AC, Gleeson J, Staughton RCD. Osteopoikilosis and the Buschke-Ollendorf syndrome. $\mathrm{Br} J$ Radiol 1993; 66:468-470.

9. Sandhu, Harvey, Ross, Robinson, Collins, Cook. Hip pain in a 38 year old man. Clin Orthop Relat Res 2004; 424:287-294.

10. Horwitz M. Osteopathia Condensans Disseminata (Osteopoikilosis). Radiology 1943; 40:404-407.

11. Agostinelli JR. Osteopoikilosis. A case report. J Am Podiary Assoc 1983; 73:529.

12. Benli IT, Akalyn S, Baysan $E$ et al. Epidemiological and radiological aspects of osteopoikilosis. J Bone Joint Surg $\mathrm{Br}$ 1992; 74(4):504-506. 
13. Lagier R, Mbakop A, Bigler A. Osteopoikilosis: a radiological and pathological study. Skeletal Radiol 1984; 11(3):161-8.

14. Greenspan A, Jundt G, Remagen W. Differential Diagnosis in Orthopaedic Oncology. Lippincott. Second Edition. Phyladelphya: Chapter 2; 2007: 51-74.

15. Young LW, Gersman I, Simon PR. Radiological case of the month. Osteopoikilosis: familial documentation. Am $J$ Dis Child 1980; 134(4):415-416.

16. Havitcioglu H, Gunal I, Gocen S. Synovial chondromatosis associated with osteopoikilosis-A case report. Acta Orthop Scand 1998; 69:649-650.

17. Alymlahi E, Chami I, Boujida NM, Bacadi D. Osteopoikilosis (OPK). Applied Radiol 2007; 36(3). 